Manuelle Medizin 2009 • 47:97-98

DOI 10.1007/s00337-009-0682-1

Online publiziert: 23. April 2009

๑) Springer Medizin Verlag 2009

\author{
L. Beyer ${ }^{1} \cdot$ R. Kayser ${ }^{2}$ \\ ${ }^{1}$ Ärztehaus Mitte, Jena \\ 2 Klink und Poliklinik für Orthopädie und Orthopädische \\ Chirurgie, Universitätsklinikum Greifswald, Greifswald
}

\title{
Qualität und Wirtschaftlichkeit
}

und sozialen Aspekten in die Schulmedizin einordnen. Dies betrifft uns aktuell und objektiv. Das Institut für Qualität und Wirtschaftlichkeit im Gesundheitswesen (IQWiG) hat am 16.03.09 den Entwurf der Methoden zur Kosten-Nutzen-Bewertung herausgegeben. Das IQWiG (http://www. iqwig.de) soll unabhängig und wissenschaftlich den Nutzen medizinischer Leistungen für Patienten untersuchen. Damit stehen Qualität und Wirtschaftlichkeit auf dem Prüfstand. Die Frage lautet, was ist therapeutisch und diagnostisch möglich und sinnvoll, als Grundlage für die Entscheidung von Kostenträgern. Hierfür werden wir uns noch besser aufstellen müssen, Unsere Zeitschrift, die Forschungsberatungsstelle der DGMM und die Kliniken für manuelle Medizin sowie größere Praxen mit Chirotherapie/osteopathischer Medizin können hier ihren Beitrag leisten. Es wird nicht mehr ausreichen, manuelle Therapie als Krankengymnastik abzurechnen oder zu formulieren: „Es wurde manuell/osteopathisch behandelt". Wann wird was und wie behandelt? - Das ist die Frage.

Neumer von der Forschungsberatungsstelle vergleicht die Entwicklung des Inhalts unserer Zeitschrift zwischen 1997 und 2007. Dabei stellt sie fest, dass sich der Schwerpunkt ein wenig in Richtung „Originalarbeiten“ verschoben hat, obwohl Beiträge mit Inhalten von „Fortund Weiterbildungscharakter" nach wie vor einen großen Stellenwert haben. Im Jahrgang 1997 gehörten 42\% aller Seiten gehören zu Originalartikeln, 2007 sind es
$49 \%$. Schön wäre es zu folgern, dass sich hier unser Bemühen auswirkt, das Fachgebiet auf ein stabiles wissenschaftliches Fundament zu stellen. In der Qualität und im wissenschaftlichen Anspruch der „Originalarbeiten“ lassen sich im Zeitverlauf aber kaum Veränderungen beobachten.

\section{Dokumentation der Behandlung und Qualitätsmanagement}

Ein Ziel der manuellen Medizin ist die Beseitigung von Funktionsstörungen im Bewegungssystem. Vor diesem Hintergrund gewinnen die Dokumentation der Behandlung und das Qualitätsmanagement für die Therapeuten der manuellen Medizin zunehmend an Bedeutung. Die Interventionskategorien des ICF (Internationale Klassifikation der Funktionsfähigkeit, Behinderung und Gesundheit) als ein Rahmenkonzept zur Beschreibung der funktionalen Gesundheit von Patienten könnten als hilfreiches Instrument in die Dokumentation und in das Qualitätsmanagementsystem integriert werden. Ziel der ICF-Klassifikation ist eine einheitliche und standardisierte Sprache zur Beschreibung von Gesundheitsstörungen. Unsere Leserinnen und Leser können sich hier einbringen. Erste Ergebnisse in diesem Heft: „ICF-Interventionskategorien für manuelle Medizin“ - ein erster Beitrag zur standardisierten Therapiedokumentation und Erfolgskontrolle" aus der Arbeitsgruppe von Prof. Stucki, München, in Zusammenarbeit mit der Schweizerischen Ärztegesellschaft beachten haben. Die manuelle Medizin muss sich auch unter wirtschaftlichen 
für Manuelle Medizin (SAMM). Die erste Version der „ICF-Interventionskategorien für manuelle Medizin“ liegt damit vor und bedarf weiterer Validierung. Der Beitrag von Ammer aus der Literatursichtung ergänzt dieses Thema mit einer Studie australischer Physiotherapeuten: „Was verstehen Rückenschmerzpatienten unter dem Begriff, wiederhergestellt?““

Wie sich manualmedizinisches Gedankengut in klinische Fachgebiete integriert, zeigen die Beiträge von Mengemann (HNO) und Gorzny (Ophthalmologie) mit Aussagen wie: „Der interdisziplinäre Ansatz der manuellen Medizin bietet das Zusammenführen von multiplen diagnostischen Ergebnissen und therapeutischen Möglichkeiten“ und "Schlüsselregionen bestimmen das manuelle diagnostische Vorgehen und den Behandlungsplan in einem dynamischen Wirkgefüge."

Veröffentlichte Beiträge sollen zumindest in Relation zum Gegenstand der manuellen Medizin stehen.
Eine Studie von Swinarek liefert Hinweise, dass ein Ganzkörpervibrationstraining für ältere Menschen als adäquates Training zur Verbesserung der Rumpfmuskulatur und der Gleichgewichtsfähigkeit angesehen werden kann und somit Alltagsmotorik und Haltung fördert.

Es gibt gute Gründe für manuell Untersuchende und Behandelnde - Ärzte und Physiotherapeuten - optimistisch zu sein. Diesem Optimismus sollte auch das aktive Mitwirken an den Zielen unserer Zeitschrift entspringen.

Wir wünschen Ihnen eine gute Frühlingszeit.

Ihre

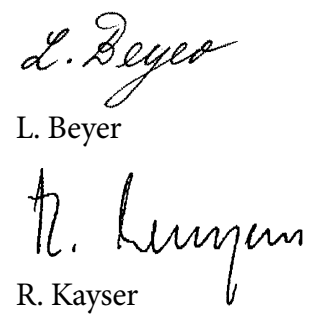

\section{Korrespondenzadresse}

Prof. Dr. L. Beyer

Ärztehaus Mitte

Westbahnhofstraße 2, 07745 Jena

lobeyer@t-online.de

\section{R. Kayser}

Klink und Poliklinik für Orthopädie und Orthopädische Chirurgie, Universitätsklinikum Greifswald Ferdinand-Sauerbruch-Straße, 17475 Greifswald

ralph.kayser@uni-greifswald.de

\section{Hier steht eine Anzeige.}

\section{望 Springer}

